Research Article

\title{
General Iterative Methods for System of Equilibrium Problems and Constrained Convex Minimization Problem in Hilbert Spaces
}

\author{
Peichao Duan \\ College of Science, Civil Aviation University of China, Tianjin 300300, China \\ Correspondence should be addressed to Peichao Duan; pcduancauc@126.com
}

Received 29 December 2012; Accepted 12 July 2013

Academic Editor: Luigi Muglia

Copyright @ 2013 Peichao Duan. This is an open access article distributed under the Creative Commons Attribution License, which permits unrestricted use, distribution, and reproduction in any medium, provided the original work is properly cited.

We propose an implicit iterative scheme and an explicit iterative scheme for finding a common element of the set of solutions of system of equilibrium problems and a constrained convex minimization problem by the general iterative methods. In the setting of real Hilbert spaces, strong convergence theorems are proved. Our results improve and extend the corresponding results reported by Tian and Liu (2012) and many others. Furthermore, we give numerical example to demonstrate the effectiveness of our iterative scheme.

\section{Introduction}

Let $H$ be a real Hilbert space with inner product $\langle$,$\rangle and$ induced norm $\|\cdot\|$. Let $C$ be a nonempty closed convex subset of $H$.

Let $\left\{F_{k}\right\}$ be a countable family of bifunctions from $C \times$ $C$ to $\mathbb{R}$, where $\mathbb{R}$ is the set of real numbers. Combettes and Hirstoaga [1] considered the following system of equilibrium problems which is to find $x \in C$ such that

$$
F_{k}(x, y) \geq 0, \quad \forall k \in \Gamma, \forall y \in C,
$$

where $\Gamma$ is an arbitrary index set. If $\Gamma$ is a singleton, then problem (1) becomes the following equilibrium problem:

$$
\text { finding } x \in C \text { such that } F(x, y) \geq 0, \quad \forall y \in C \text {. }
$$

The solution set of (2) is denoted by $\mathrm{EP}(F)$.

Numerous problems in physics, optimization, and economics reduce to finding a solution of the equilibrium problem. Many methods have been proposed to solve the equilibrium problem (2); see [2-4] and the references therein. In particular, some methods have been proposed to solve the system of equilibrium problems. See [5-7] and the references therein.
On the other hand, we consider the following constrained minimization problem:

$$
\operatorname{minimize}\{f(x): x \in C\},
$$

where $f: C \rightarrow \mathbb{R}$ is a real-valued convex function. It is known that the gradient projection algorithm (GPA) is a powerful tool for solving the constrained minimization problems and has extensively been studied; see for instance [8-10]. If $f$ is (Fréchet) differentiable, then the GPA generates a sequence $\left\{x_{n}\right\}$ using the following recursive formula:

$$
x_{n+1}=P_{C}\left(x_{n}-\lambda \nabla f\left(x_{n}\right)\right), \quad \forall n \geq 0,
$$

or more generally,

$$
x_{n+1}=P_{C}\left(x_{n}-\lambda_{n} \nabla f\left(x_{n}\right)\right), \quad \forall n \geq 0,
$$

where in both (4) and (5) the initial guess $x_{0}$ is taken from $C$ arbitrarily, and the parameters, $\lambda$ or $\lambda_{n}$, are positive real numbers satisfying certain conditions. The convergence of the algorithms (4) and (5) depends on the behavior of the gradient $\nabla f$. As a matter of fact, it is known that if $\nabla f$ is $\alpha$-strongly monotone and $L$-Lipschitzian with constants $\alpha$, $L \geq 0$, then the operator

$$
T:=P_{C}(I-\lambda \nabla f)
$$


is a contraction; hence, the sequence $\left\{x_{n}\right\}$ defined by the algorithm (4) converges in norm to the unique minimizer of (3). However, if the gradient $\nabla f$ fails to be strongly monotone, the operator $T$ by (6) would fail to be contractive; consequently, the sequence $\left\{x_{n}\right\}$ generated by the algorithm (4) may fail to converge strongly [11]. If $\nabla f$ is Lipschitzian, then the algorithms (4) and (5) can still converge in the weak topology under certain conditions $[10,12]$.

In 2007, Marino and Xu [3] introduced the general iterative method and proved that the algorithm converged strongly. In 2009, Liu [2] considered two iterative schemes by the general iterative method for equilibrium problems and strict pseudocontractions. In 2011, Xu [11] gave an alternative operator-oriented approach to algorithm (5), namely, an averaged mapping approach. He gave his averaged mapping approach to the GPA (5) and the relaxed GPA. Moreover, he constructed a counter example which shows that the algorithm (4) does not converge in norm in an infinite-dimensional space and also presented two modifications of GPA which are shown to have strong convergence. Recently, Ceng et al. [8] proposed implicit and explicit iterative schemes for finding the approximate minimizer of a constrained convex minimization problem and proved that the sequences generated by their schemes converges strongly to a solution of the constrained convex minimization problem. Very recently, Tian and Liu [9] proposed implicit and explicit composite iterative algorithms for finding a common solution of an equilibrium problem and a constrained convex minimization problem; strong convergence theorems are obtained in [9].

In this paper, motivated by the above facts, we introduce two iterative schemes by the composite general iterative methods. Further we obtain strong convergence theorems for finding a common element of the set of solutions of a constrained convex minimization problems and the set of solutions of the equilibrium problem

$$
F_{k}(x, y) \geq 0, \quad \forall k \in \Gamma, \forall y \in C,
$$

where $\Gamma=\{1,2, \ldots, M\}$ is a finite index set.

\section{Preliminaries}

Throughout this paper, we always write $\rightarrow$ for weak convergence and $\rightarrow$ for strong convergence. We need some definitions and tools in a real Hilbert space $H$ which are listed as below.

A mapping $S$ of $C$ is said to be a nonexpansive mapping such that

$$
\|S x-S y\| \leq\|x-y\|
$$

for all $x, y \in C$. The set of fixed points of $S$ is denoted by $F(S)$; that is, $F(S)=\{x \in C: S x=x\}$.

A mapping $T: H \rightarrow H$ is said to be an averaged mapping if it can be written as the average of an identity $I$ and a nonexpansive mapping; that is,

$$
T=(1-\alpha) I+\alpha S,
$$

where $\alpha$ is a number in $(0,1)$ and $S$ is nonexpansive. More precisely, we say that $T$ is $\alpha$-averaged. It is known that the projection is $(1 / 2)$-averaged.
Lemma 1. Let $H$ be a real Hilbert space. There hold the following identities:

(i) $\|x-y\|^{2}=\|x\|^{2}-\|y\|^{2}-2\langle x-y, y\rangle, \forall x, y \in H$,

(ii) $\|t x+(1-t) y\|^{2}=t\|x\|^{2}+(1-t)\|y\|^{2}-t(1-t)\|x-y\|^{2}$, $\forall t \in[0,1], \forall x, y \in H$.

Lemma 2 (see $[10])$. Assume that $\left\{\alpha_{n}\right\}$ is a sequence of nonnegative real numbers such that

$$
\alpha_{n+1} \leq\left(1-\gamma_{n}\right) \alpha_{n}+\delta_{n},
$$

where $\left\{\gamma_{n}\right\}$ is a sequence in $(0,1)$ and $\left\{\delta_{n}\right\}$ is a sequence such that

(i) $\sum_{n=1}^{\infty} \gamma_{n}=\infty$;

(ii) $\lim \sup _{n \rightarrow \infty} \delta_{n} / \gamma_{n} \leq 0$ or $\sum_{n=1}^{\infty}\left|\delta_{n}\right|<\infty$.

Then, $\lim _{n \rightarrow \infty} \alpha_{n}=0$.

Recall that given a nonempty closed convex subset $C$ of a real Hilbert space $H$, for any $x \in H$, there exists a unique nearest point in $C$, denoted by $P_{C} x$, such that

$$
\left\|x-P_{C} x\right\| \leq\|x-y\|
$$

for all $y \in C$. Such a $P_{C}$ is called the metric (or the nearest point) projection of $H$ onto $C$. As we all know, $y=P_{C} x$ if and only if there holds the relation:

$$
\langle x-y, y-z\rangle \geq 0, \quad \forall z \in C .
$$

Lemma 3 (see [13]). Let $A: H \rightarrow H$ be an L-Lipschitzian and $\eta$-strongly monotone operator on a Hilbert space $H$ with $L>0, \eta>0,0<\mu<2 \eta / L^{2}$ and $0<t<1$. Then $S=(I-t \mu A):$ $H \rightarrow H$ is a contraction with contractive coefficient $1-t \tau$ and $\tau=(1 / 2) \mu\left(2 \eta-\mu L^{2}\right)$.

Definition 4. A nonlinear mapping $A$ whose domain $D(A) \subseteq$ $H$ and range $R(A) \subseteq H$ is said to be

(i) monotone if

$$
\langle A x-A y, x-y\rangle \geq 0, \quad \forall x, y \in D(A) ;
$$

(ii) $\beta$-strongly monotone if there exists $\beta>0$ such that

$$
\langle A x-A y, x-y\rangle \geq \beta\|x-y\|^{2}, \quad \forall x, y \in D(A) ;
$$

(iii) $\nu$-inverse strongly monotone (for short, $\nu$-ism) if there exists a constant $v>0$ such that

$$
\langle A x-A y, x-y\rangle \geq v\|A x-A y\|^{2}, \quad \forall x, y \in D(A) .
$$

Lemma 5. Let $V: C \rightarrow H$ be an l-Lipschitz mapping with coefficient $l \geq 0$ and $A: C \rightarrow H$ a strong positive bounded linear operator with $\bar{\gamma}>0$. Then for $0<\gamma<\mu \bar{\gamma} / l$,

$$
\begin{array}{r}
\langle x-y,(\mu A-\gamma V) x-(\mu A-\gamma V) y\rangle \\
\geq(\mu \bar{\gamma}-\gamma l)\|x-y\|^{2}, \quad x, y \in H ;
\end{array}
$$

that is, $\mu A-\gamma V$ is strongly monotone with coefficient $\mu \bar{\gamma}-\gamma l$. 
Proof. Since $A: C \rightarrow H$ is a strong positive bounded linear operator with $\bar{\gamma}>0$. We have

$$
\begin{aligned}
\bar{\gamma}\|x-y\|^{2} & \leq\langle x-y, A x-A y\rangle \\
& =\langle x-y, A(x-y)\rangle \leq\|A\|\|x-y\|^{2} .
\end{aligned}
$$

Hence $A$ is $\|A\|$-Lipschitz and $\bar{\gamma}$-strongly monotone:

$$
\begin{aligned}
& \langle x-y,(\mu A-\gamma V) x-(\mu A-\gamma V) y\rangle \\
& =\mu\langle x-y, A x-A y\rangle-\gamma\langle x-y, V x-V y\rangle \\
& \geq(\mu \bar{\gamma}-\gamma l)\|x-y\|^{2}, \quad x, y \in H .
\end{aligned}
$$

Proposition 6. For given operators $S, T, V: H \rightarrow H$.

(i) If $T=(1-\alpha) S+\alpha V$ for some $\alpha \in(0,1)$ and if $S$ is averaged and $V$ is nonexpansive, then $T$ is averaged.

(ii) $T$ is firmly nonexpansive if and only if the complement $I-T$ is firmly nonexpansive.

(iii) If $T=(1-\alpha) S+\alpha V$ for some $\alpha \in(0,1), S$ is firmly nonexpansive, and $V$ is nonexpansive, then $T$ is averaged.

(iv) The composite of finitely many averaged mappings is averaged; that is, if each of the mapping $\left\{T_{i}\right\}_{i=1}^{N}$ is averaged, then so is the composite $T_{1}, \ldots, T_{N}$. In particular, if $T_{1}$ is $\alpha_{1}$-averaged and $T_{2}$ is $\alpha_{2}$-averaged, where $\alpha_{1}, \alpha_{2} \in(0,1)$, then the composite $T_{1} T_{2}$ is $\alpha$ averaged, where $\alpha=\alpha_{1}+\alpha_{2}-\alpha_{1} \alpha_{2}$.

Proposition 7. Let $T: H \rightarrow H$ be an operator from $H$ to itself.

(i) $T$ is nonexpansive if and only if the complement $I-T$ is $(1 / 2)-i s m$.

(ii) If T is $\nu$-ism, then for $\gamma>0, \gamma T$ is $(\nu / \gamma)$-ism.

(iii) $T$ is averaged if and only if the complement $I-T$ is $\nu$-ism for some $\nu>(1 / 2)$. Indeed, for $\alpha \in(0,1), T$ is $\alpha$-averaged if and only if the complement $I-T$ is $(1 / 2 \alpha)$-ism

For solving the equilibrium problem, let us assume that the bifunction $F$ satisfies the following conditions.

(A1) $F(x, x)=0$ for all $x \in C$.

(A2) $F$ is monotone; that is, $F(x, y)+F(y, x) \leq 0$ for any $x, y \in C$;

(A3) For each $x, y, z \in C$, $\limsup _{t \rightarrow 0} F(t z+(1-t) x, y) \leq$ $F(x, y)$.

(A4) $F(x, \cdot)$ is convex and lower semicontinuous for each $x \in C$.

We recall some lemmas which will be needed in the rest of this paper.
Lemma 8 (see [14]). Let $C$ be a nonempty closed convex subset of $H$, let $F$ be bifunction from $C \times C$ to $\mathbb{R}$ satisfying (A1)-(A4), and let $r>0$ and $x \in H$. Then there exists $z \in C$ such that

$$
F(z, y)+\frac{1}{r}\langle y-z, z-x\rangle \geq 0, \quad \forall y \in C .
$$

Lemma 9 (see [1]). For $r>0, x \in H$, define a mapping $T_{r}$ : $H \rightarrow C$ as follows:

$$
T_{r}(x)=\left\{z \in C \mid F(z, y)+\frac{1}{r}\langle y-z, z-x\rangle \geq 0, \quad \forall y \in C\right\}
$$

for all $x \in H$. Then, the following statements hold:

(i) $T_{r}$ is single-valued;

(ii) $T_{r}$ is firmly nonexpansive; that is, for any $x, y \in H$,

$$
\left\|T_{r} x-T_{r} y\right\|^{2} \leq\left\langle T_{r} x-T_{r} y, x-y\right\rangle ;
$$

(iii) $F\left(T_{r}\right)=\operatorname{EP}(F)$;

(iv) $\mathrm{EP}(F)$ is closed and convex.

Lemma 10 (see [4]). Let $C, H, F$, and $T_{r} x$ be as in Lemma 9. Then the following holds:

$$
\left\|T_{s} x-T_{t} x\right\|^{2} \leq \frac{s-t}{s}\left\langle T_{s} x-T_{t} x, T_{s} x-x\right\rangle
$$

for all $s, t>0$ and $x \in H$.

Lemma 11 (see [13]). Let $H$ be a Hilbert space, $C$ a nonempty closed convex subset of $H$, and $T: C \rightarrow C$ a nonexpansive mapping with $F(T) \neq \emptyset$. If $\left\{x_{n}\right\}$ is a sequence in $C$ weakly converging to $x$ and if $\left\{(I-T) x_{n}\right\}$ converges strongly to $y$, then $(I-T) x=y$.

\section{Main Result}

Throughout the rest of this paper, we always assume that $V$ is an $l$-Lipschitzian mapping with coefficient $l \geq 0$, and $A$ is a strongly positive bounded linear operator with coefficient $\bar{\gamma}$. Then we obtain that $A$ is $\|A\|$-Lipschitzian and $\bar{\gamma}$-strongly monotone. Let $f: C \rightarrow R$ be a real-valued convex function and assume that $\nabla f$ is $\nu$-ism with $v>0$, which then implies that $\lambda \nabla f$ is $\nu / \lambda$-ism. So by Proposition 7 , its complement $I-\lambda \nabla f$ is $\lambda /(2 \nu)$-averaged. Since the projection $P_{C}$ is $1 / 2$ averaged, we obtain from Proposition 6 that the composition $P_{C}(I-\lambda \nabla f)$ is $(2 \nu+\lambda) /(4 \nu)$-averaged for $0<\lambda<2 / L$. Hence we have that, for each $n, P_{C}\left(I-\lambda_{n} \nabla f\right)$ is $\left(2 \nu+\lambda_{n}\right) /(4 \nu)$ averaged. Therefore, we can write

$$
P_{C}\left(I-\lambda_{n} \nabla f\right)=\frac{2 v-\lambda_{n}}{4 v} I+\frac{2 v+\lambda_{n}}{4 v} S_{n}
$$

where $S_{n}$ is nonexpansive.

Suppose that the minimization problem (3) is consistent and let $U$ denote its solution set. Assume that $0<\mu<$ $2 \bar{\gamma} /\|A\|^{2}$ and $0<\gamma<\mu\left(\bar{\gamma}-\left(\mu\|A\|^{2} / 2\right)\right) / l=\tau / l$. 
Denote $\Theta_{n}^{k}=T_{r_{k, n}}^{F_{k}} \ldots T_{r_{2, n}}^{F_{2}} T_{r_{1, n}}^{F_{1}}$ for every $k \in\{1,2, \ldots, M\}$ and $\Theta_{n}^{0}=I$ for all $n \in \mathbb{N}$. Define a mapping $W_{n}=S_{n} \Theta_{n}^{M}$. Since both $S_{n}$ and $T_{r_{k, n}}^{F_{k}}, k \in\{1,2, \ldots, M\}$ are nonexpansive, it is easy to get that $W_{n}$ is also nonexpansive. Consider the following mapping $G_{n}$ on $H$ defined by

$$
G_{n} x=\alpha_{n} \gamma V \Theta_{n}^{M} x+\left(I-\alpha_{n} \mu A\right) W_{n} x, \quad \forall x \in H, n \in N,
$$

where $\alpha_{n} \in(0,1)$. By Lemmas 3 and 9 , we have

$$
\begin{aligned}
& \left\|G_{n} x-G_{n} y\right\| \\
& \quad \leq \alpha_{n} \gamma\|V x-V y\|+\left(1-\alpha_{n} \tau\right)\left\|W_{n} x-W_{n} y\right\| \\
& \quad \leq \alpha_{n} \gamma l\|x-y\|+\left(1-\alpha_{n} \tau\right)\|x-y\| \\
& \quad=\left(1-\alpha_{n}(\tau-\gamma l)\right)\|x-y\| .
\end{aligned}
$$

Since $0<1-\alpha_{n}(\tau-\gamma l)<1$, it follows that $G_{n}$ is a contraction. Therefore, by the Banach contraction principle, $G_{n}$ has a unique fixed pointed $x_{n}^{V} \in H$ such that

$$
x_{n}^{V}=\alpha_{n} \gamma V \Theta_{n}^{M}\left(x_{n}^{V}\right)+\left(I-\alpha_{n} \mu A\right) W_{n} x_{n}^{V} .
$$

For simplicity, we will write $x_{n}$ for $x_{n}^{V}$ provided no confusion occurs. Next we prove the sequences $\left\{x_{n}\right\}$ converges strongly to a point $x^{*} \in \Omega=U \cap \cap_{k=1}^{M} \operatorname{EP}\left(F_{k}\right)$ which solves the variational inequality

$$
\left\langle(\gamma V-\mu A) x^{*}, p-x^{*}\right\rangle \leq 0, \quad \forall p \in \Omega .
$$

Equivalently, $x^{*}=P_{\Omega}(I-\mu A+\gamma V) x^{*}$.

Theorem 12. Let $C$ be a nonempty closed convex subset of a real Hilbert space $H$ with $C \pm C \subseteq C$, and let $F_{k}, k \in$ $\{1,2, \ldots M\}$ be bifunctions from $C \times C$ to $\mathbb{R}$ satisfying (A1)(A4). Let $f: C \rightarrow \mathbb{R}$ be a real-value convex function and $\nabla f \nu$-ism with $\nu>0$. Assume the set $\Omega=U \cap \cap_{k=1}^{M} \operatorname{EP}\left(F_{k}\right) \neq \emptyset$. Let $V: C \rightarrow C$ be an l-Lipschitzian mapping with $l \geq 0$ and A a strongly positive bounded linear operator with coefficient $\bar{\gamma} \geq 0,0<\mu<2 \bar{\gamma} /\|A\|^{2}$ and $0<\gamma<\mu\left(\bar{\gamma}-\left(\mu\|A\|^{2} / 2\right)\right) / l=$ $\tau / l$. Let $\left\{x_{n}\right\}$ and $\left\{u_{n}\right\}$ be sequences generated by the following algorithm:

$$
\begin{gathered}
u_{n}=T_{r_{M, n}}^{F_{M}} T_{r_{M-1, n}}^{F_{M-1}} \cdots T_{r_{2, n}}^{F_{2}} T_{r_{1, n}}^{F_{1}} x_{n}, \\
x_{n}=\alpha_{n} \gamma V u_{n}+\left(I-\mu \alpha_{n} A\right) S_{n} u_{n},
\end{gathered}
$$

where $P_{C}\left(I-\lambda_{n} \nabla f\right)=\beta_{n} I+\left(1-\beta_{n}\right) S_{n}, \beta_{n}=\left(\left(2 \nu-\lambda_{n}\right) / 4 \nu\right)$, and $\lambda_{n} \in(0,2 \nu)$; if $\left\{\alpha_{n}\right\}$ and $\left\{r_{k, n}\right\}$ satisfy the following conditions:

(i) $\left\{\alpha_{n}\right\} \subset(0,1), \lim _{n \rightarrow \infty} \alpha_{n}=0$;

(ii) $\left\{r_{k, n}\right\} \subset(0, \infty), \liminf _{n \rightarrow \infty} r_{k, n}>0$ for $k \in\{1,2, \ldots$, $M\}$,

then, as $\beta_{n} \rightarrow 0\left(\lambda_{n} \rightarrow 2 v\right)$, the sequence $\left\{x_{n}\right\}$ converges strongly to a point $x^{*} \in \Omega$, which solves the variational inequality (27).
Proof. The proof is divided into several steps.

Step 1. It shows first that $\left\{x_{n}\right\}$ is bounded.

First, since $\lim _{n \rightarrow \infty} \alpha_{n}=0$, we can assume that $\alpha_{n} \in$ $\left(0,\|A\|^{-1}\right)$. By Lemma 3 , we have $\left\|I-\alpha_{n} \mu A\right\| \leq 1-\alpha_{n} \tau$.

Take any $p \in \Omega$, since for each $k \in\{1,2, \ldots, M\}, T_{r_{k, n}}^{F_{k}}$ is nonexpansive, $p=T_{r_{k, n}}^{F_{k}} p$, and $u_{n}=\Theta_{n}^{M} x_{n}$; we have

$$
\left\|u_{n}-p\right\|=\left\|\Theta_{n}^{M} x_{n}-\Theta_{n}^{M} p\right\| \leq\left\|x_{n}-p\right\|
$$

for all $n \in N$.

Thus, by (28) and Lemma 3, we derive that

$$
\begin{aligned}
& \left\|x_{n}-p\right\| \\
= & \left\|\alpha_{n} \gamma V u_{n}+\left(I-\mu \alpha_{n} A\right) S_{n} u_{n}-p\right\| \\
= & \|\left(I-\mu \alpha_{n} A\right) S_{n} u_{n}-\left(I-\mu \alpha_{n} A\right) S_{n} p \\
& +\alpha_{n} \gamma V u_{n}-\alpha_{n} \gamma V p+\alpha_{n} \gamma V p-\alpha_{n} \mu A p \| \\
\leq & \left(1-\alpha_{n} \tau\right)\left\|u_{n}-p\right\|+\alpha_{n} \gamma l\left\|u_{n}-p\right\|+\alpha_{n}\|(\gamma V-\mu A) p\| \\
\leq & \left(1-\alpha_{n}(\tau-\gamma l)\right)\left\|x_{n}-p\right\|+\alpha_{n}\|(\gamma V-\mu A) p\| .
\end{aligned}
$$

It follows that $\left\|x_{n}-p\right\| \leq(\|(\gamma V-\mu A) p\| /(\tau-\gamma l))$.

Hence, $\left\{x_{n}\right\}$ is bounded and so $\left\{u_{n}\right\}$. It follows from the Lipschitz continuity of $A, \nabla f$, and $V$ that $\left\{A x_{n}\right\},\left\{A u_{n}\right\}$, $\left\{\nabla f\left(u_{n}\right)\right\}$ and $\left\{V u_{n}\right\}$ are also bounded. From the nonexpansivity of $S_{n}$, it follows that $\left\{S_{n} u_{n}\right\}$ is also bounded.

Step 2. It shows that

$$
\lim _{n \rightarrow \infty}\left\|u_{n}-x_{n}\right\|=0 .
$$

Next we will show that

$$
\lim _{n \rightarrow \infty}\left\|\Theta_{n}^{k} x_{n}-\Theta_{n}^{k-1} x_{n}\right\|=0, \quad k=1,2, \ldots, M .
$$

Indeed, for $p \in \Omega$, it follows from the firmly nonexpansivity of $T_{r_{k, n}}^{F_{k}}$ that for each $k \in\{1,2, \ldots, M\}$, we have

$$
\begin{aligned}
& \left\|\Theta_{n}^{k} x_{n}-p\right\|^{2} \\
& =\left\|T_{r_{k, n}}^{F_{k}} \Theta_{n}^{k-1} x_{n}-T_{r_{k, n}}^{F_{k}} p\right\|^{2} \\
& \leq\left\langle\Theta_{n}^{k} x_{n}-p, \Theta_{n}^{k-1} x_{n}-p\right\rangle \\
& =\frac{1}{2}\left(\left\|\Theta_{n}^{k} x_{n}-p\right\|^{2}+\left\|\Theta_{n}^{k-1} x_{n}-p\right\|^{2}\right. \\
& \left.\quad-\left\|\Theta_{n}^{k} x_{n}-\Theta_{n}^{k-1} x_{n}\right\|^{2}\right) .
\end{aligned}
$$

Thus we get

$$
\begin{aligned}
\left\|\Theta_{n}^{k} x_{n}-p\right\|^{2} \leq & \left\|\Theta_{n}^{k-1} x_{n}-p\right\|^{2} \\
& -\left\|\Theta_{n}^{k} x_{n}-\Theta_{n}^{k-1} x_{n}\right\|^{2}, \quad k=1,2, \ldots, M,
\end{aligned}
$$


which implies that for each $k \in\{1,2, \ldots, M\}$,

$$
\begin{aligned}
& \left\|\Theta_{n}^{k} x_{n}-p\right\|^{2} \\
& \leq\left\|\Theta_{n}^{0} x_{n}-p\right\|^{2}-\left\|\Theta_{n}^{k} x_{n}-\Theta_{n}^{k-1} x_{n}\right\|^{2} \\
& \quad-\left\|\Theta_{n}^{k-1} x_{n}-\Theta_{n}^{k-2} x_{n}\right\|^{2} \cdots-\left\|\Theta_{n}^{1} x_{n}-\Theta_{n}^{0} x_{n}\right\|^{2} \\
& \leq\left\|x_{n}-p\right\|^{2}-\left\|\Theta_{n}^{k} x_{n}-\Theta_{n}^{k-1} x_{n}\right\|^{2} .
\end{aligned}
$$

Thus, from Lemma 1 and (35), we get

$$
\begin{aligned}
\left\|x_{n}-p\right\|^{2}= & \left\|\alpha_{n} \gamma V u_{n}+\left(I-\mu \alpha_{n} A\right) S_{n} u_{n}-p\right\|^{2} \\
= & \|\left(I-\mu \alpha_{n} A\right) S_{n} u_{n}-\left(I-\mu \alpha_{n} A\right) S_{n} p \\
& +\alpha_{n} \gamma V u_{n}-\alpha_{n} \mu A p \|^{2} \\
\leq & \left(1-\alpha_{n} \tau\right)^{2}\left\|u_{n}-p\right\|^{2} \\
& +2 \alpha_{n}\left\langle\gamma V u_{n}-\mu A p, x_{n}-p\right\rangle \\
\leq & \left(1-\alpha_{n} \tau\right)^{2}\left\|u_{n}-p\right\|^{2} \\
& +2 \alpha_{n}\left\langle\gamma V u_{n}-\gamma V p+\gamma V p-\mu A p, x_{n}-p\right\rangle \\
\leq & \left(1-\alpha_{n} \tau\right)^{2}\left(\left\|x_{n}-p\right\|^{2}-\left\|\Theta_{n}^{k} x_{n}-\Theta_{n}^{k-1} x_{n}\right\|^{2}\right) \\
& +2 \alpha_{n} \gamma l\left\|x_{n}-p\right\|^{2}+2 \alpha_{n}\|\gamma V p-\mu A p\|\left\|x_{n}-p\right\| \\
\leq & \left\|x_{n}-p\right\|^{2}+\left(\alpha_{n} \tau\right)^{2}\left\|x_{n}-p\right\|^{2} \\
& -\left(1-\alpha_{n} \tau\right)^{2}\left\|\Theta_{n}^{k} x_{n}-\Theta_{n}^{k-1} x_{n}\right\|^{2} \\
& +2 \alpha_{n}\|\gamma V p-\mu A p\|\left\|x_{n}-p\right\| .
\end{aligned}
$$

It follows that

$$
\begin{aligned}
& \left(1-\alpha_{n} \tau\right)^{2}\left\|\Theta_{n}^{k} x_{n}-\Theta_{n}^{k-1} x_{n}\right\|^{2} \\
& \quad \leq\left(\alpha_{n} \tau\right)^{2}\left\|x_{n}-p\right\|^{2}+2 \alpha_{n}\|\gamma V p-\mu A p\|\left\|x_{n}-p\right\| .
\end{aligned}
$$

Since $\alpha_{n} \rightarrow 0$, (32) holds, then we have

$$
\begin{aligned}
\left\|u_{n}-x_{n}\right\| \leq & \left\|u_{n}-\Theta_{n}^{M-1} x_{n}\right\| \\
& +\left\|\Theta_{n}^{M-1} x_{n}-\Theta_{n}^{M-2} x_{n}\right\| \\
& +\cdots+\left\|\Theta_{n}^{1} x_{n}-x_{n}\right\| \longrightarrow 0 .
\end{aligned}
$$

Step 3. It shows that

$$
\lim _{n \rightarrow \infty}\left\|x_{n}-S_{n} x_{n}\right\|=0 .
$$

Observe that

$$
\begin{aligned}
\| x_{n}- & S_{n} x_{n} \| \\
& =\left\|x_{n}-S_{n} u_{n}+S_{n} u_{n}-S_{n} x_{n}\right\| \\
& \leq\left\|x_{n}-S_{n} u_{n}\right\|+\left\|S_{n} u_{n}-S_{n} x_{n}\right\| \\
& \leq \alpha_{n}\left\|\gamma V u_{n}-\mu A S_{n} u_{n}\right\|+\left\|u_{n}-x_{n}\right\| .
\end{aligned}
$$

Since $\alpha_{n} \rightarrow 0$ and $\left\|u_{n}-x_{n}\right\| \rightarrow 0$, it is easy to get (39).
Thus,

$$
\begin{aligned}
\| u_{n} & -S_{n} u_{n} \| \\
& =\left\|u_{n}-x_{n}+x_{n}-S_{n} x_{n}+S_{n} x_{n}-S_{n} u_{n}\right\| \\
& \leq 2\left\|x_{n}-u_{n}\right\|+\left\|x_{n}-S_{n} x_{n}\right\| .
\end{aligned}
$$

We obtain $\left\|u_{n}-S_{n} u_{n}\right\| \rightarrow 0$.

Notice that

$$
\begin{aligned}
\| P_{C} & \left(I-\lambda_{n} \nabla f\right) u_{n}-u_{n} \| \\
& =\left\|\beta_{n} u_{n}+\left(1-\beta_{n}\right) S_{n} u_{n}-u_{n}\right\| \\
& =\left(1-\beta_{n}\right)\left\|u_{n}-S_{n} u_{n}\right\| \\
& \leq\left\|u_{n}-S_{n} u_{n}\right\|,
\end{aligned}
$$

where $\beta_{n} \in(0,1 / 2)$. Hence we have

$$
\begin{aligned}
&\left\|P_{C}(I-2 \nu \nabla f) u_{n}-u_{n}\right\| \\
& \leq\left\|P_{C}(I-2 \nu \nabla f) u_{n}-P_{C}\left(I-\lambda_{n} \nabla f\right) u_{n}\right\| \\
&+\left\|P_{C}\left(I-\lambda_{n} \nabla f\right) u_{n}-u_{n}\right\| \\
& \leq\left\|(I-2 \nu \nabla f) u_{n}-\left(I-\lambda_{n} \nabla f\right) u_{n}\right\| \\
&+\left\|P_{C}\left(I-\lambda_{n} \nabla f\right) u_{n}-u_{n}\right\| \\
& \leq\left(2 \nu-\lambda_{n}\right)\left\|\nabla f\left(u_{n}\right)\right\|+\left\|S_{n} u_{n}-u_{n}\right\| .
\end{aligned}
$$

From the boundedness of $\left\{\nabla f\left(u_{n}\right)\right\}, \beta_{n} \rightarrow 0$ and $\| S_{n} u_{n}-$ $u_{n} \| \rightarrow 0$, we conclude that

$$
\lim _{n \rightarrow \infty}\left\|P_{C}(I-2 \nu \nabla f) u_{n}-u_{n}\right\|=0 .
$$

Since $\left\{u_{n}\right\}$ is bounded, there exists a subsequence $\left\{u_{n_{j}}\right\}$ which converges weakly to $x^{*}$.

Step 4. It shows that $x^{*} \in \Omega$.

Since $C$ is closed and convex, $C$ is weakly closed. So we have $x^{*} \in C$. By Lemma 11 and (44), we have $x^{*} \in U$.

Next we will show that $x^{*} \in \cap_{i=1}^{M} \operatorname{EP}\left(F_{k}\right)$.

Indeed, by Lemma 9, we have that for each $k=1,2$, ..., $M$,

$$
F_{k}\left(\Theta_{n}^{k} x_{n}, y\right)+\frac{1}{r_{k, n}}\left\langle y-\Theta_{n}^{k} x_{n}, \Theta_{n}^{k} x_{n}-\Theta_{n}^{k-1} x_{n}\right\rangle \geq 0,
$$

$\forall y \in C$.

From (A2), we get

$$
\frac{1}{r_{k, n}}\left\langle y-\Theta_{n}^{k} x_{n}, \Theta_{n}^{k} x_{n}-\Theta_{n}^{k-1} x_{n}\right\rangle \geq F_{k}\left(y, \Theta_{n}^{k} x_{n}\right),
$$

$\forall y \in C$.

Hence,

$$
\left\langle y-\Theta_{n_{j}}^{k} x_{n_{j}}, \frac{\Theta_{n_{j}}^{k} x_{n_{j}}-\Theta_{n_{j}}^{k-1} x_{n_{j}}}{r_{k, n_{j}}}\right\rangle \geq F_{k}\left(y, \Theta_{n_{j}}^{k} x_{n_{j}}\right),
$$

$\forall y \in C$. 
From (32), we obtain that $\Theta_{n_{j}}^{k} x_{n_{j}} \rightarrow x^{*}$ as $j \rightarrow \infty$ for each $k=1,2, \ldots, M$ (especially, $u_{n_{j}}=\Theta_{n_{j}}^{M} x_{n_{j}}$ ). Together with (32), condition (ii), and (A4) we have, for each $k=1,2, \ldots, M$, that

$$
0 \geq F_{k}\left(y, x^{*}\right), \quad \forall y \in C
$$

For any, $0<t \leq 1$ and $y \in C$, let $y_{t}=t y+(1-t) x^{*}$. Since $y \in C$ and $x^{*} \in C$, we obtain that $y_{t} \in C$, and hence $F_{k}\left(y_{t}, x^{*}\right) \leq 0$. So, we have

$$
\begin{aligned}
0 & =F_{k}\left(y_{t}, y_{t}\right) \leq t F_{k}\left(y_{t}, y\right)+(1-t) F_{k}\left(y_{t}, x^{*}\right) \\
& \leq t F_{k}\left(y_{t}, y\right) .
\end{aligned}
$$

Dividing by $t$, we get, for each $k=1,2, \ldots, M$, that

$$
F_{k}\left(y_{t}, y\right) \geq 0, \quad \forall y \in C \text {. }
$$

Letting $t \rightarrow 0$ and from (A3), we get

$$
F_{k}\left(x^{*}, y\right) \geq 0 \text {. }
$$

For all $y \in C$ and $x^{*} \in \operatorname{EP}\left(F_{k}\right)$ for each $k=1,2, \ldots, M$; that is, $x^{*} \in \cap_{k=1}^{M} \operatorname{EP}\left(F_{k}\right)$. Hence $x^{*} \in \Omega$.

Step 5. It shows that $x_{n} \rightarrow x^{*}$, where $x^{*}=P_{\Omega}(I-\mu A+\gamma V) x^{*}$

$$
\begin{aligned}
x_{n}-x^{*}= & \alpha_{n}\left(\gamma V u_{n}-\mu A x^{*}\right) \\
& +\left(I-\mu \alpha_{n} A\right) S_{n} u_{n}-\left(I-\mu \alpha_{n} A\right) x^{*} .
\end{aligned}
$$

Hence, we obtain

$$
\begin{aligned}
\| x_{n}- & x^{*} \|^{2} \\
= & \alpha_{n}\left\langle\gamma V u_{n}-\mu A x^{*}, x_{n}-x^{*}\right\rangle \\
& +\left\langle\left(I-\mu \alpha_{n} A\right) S_{n} u_{n}-\left(I-\mu \alpha_{n} A\right) x^{*}, x_{n}-x^{*}\right\rangle \\
\leq & \alpha_{n}\left\langle\gamma V u_{n}-\mu A x^{*}, x_{n}-x^{*}\right\rangle \\
& +\left(1-\alpha_{n} \tau\right)\left\|x_{n}-x^{*}\right\|^{2} .
\end{aligned}
$$

It follows that

$$
\begin{aligned}
& \left\|x_{n}-x^{*}\right\|^{2} \\
& \leq \frac{1}{\tau}\left\langle\gamma V u_{n}-\mu A x^{*}, x_{n}-x^{*}\right\rangle \\
& =\frac{1}{\tau}\left(\gamma\left\langle V u_{n}-V x^{*}, x_{n}-x^{*}\right\rangle+\left\langle\gamma V x^{*}-\mu A x^{*}, x_{n}-x^{*}\right\rangle\right) \\
& \leq \frac{1}{\tau}\left(\gamma l\left\|x_{n}-x^{*}\right\|^{2}+\left\langle\gamma V x^{*}-\mu A x^{*}, x_{n}-x^{*}\right\rangle\right) .
\end{aligned}
$$

This implies that

$$
\left\|x_{n}-x^{*}\right\|^{2} \leq \frac{\left\langle\gamma V x^{*}-\mu A x^{*}, x_{n}-x^{*}\right\rangle}{\tau-\gamma l} .
$$

In particular,

$$
\left\|x_{n_{j}}-x^{*}\right\|^{2} \leq \frac{\left\langle\gamma V x^{*}-\mu A x^{*}, x_{n_{j}}-x^{*}\right\rangle}{\tau-\gamma l} .
$$

Since $x_{n_{j}} \rightarrow x^{*}$, it follows from (56) that $x_{n_{j}} \rightarrow x^{*}$ as $j \rightarrow \infty$. Next, we show that $x^{*}$ solves the variational inequality (27).

By the iterative algorithm (28), we have

$$
x_{n}=\alpha_{n} \gamma V u_{n}+\left(I-\mu \alpha_{n} A\right) S_{n} u_{n} .
$$

Therefore we have

$$
\begin{aligned}
\alpha_{n}(\mu A-\gamma V) x_{n} \\
=\alpha_{n}(\mu A-\gamma V)\left(I-W_{n}\right) x_{n}+\alpha_{n}(\mu A-\gamma V) S_{n} u_{n} \\
\quad+\alpha_{n} \gamma V u_{n}+\left(I-\mu \alpha_{n} A\right) S_{n} u_{n}-x_{n} \\
=\alpha_{n}(\mu A-\gamma V)\left(I-W_{n}\right) x_{n} \\
\quad+\alpha_{n} \gamma\left(V u_{n}-V S_{n} u_{n}\right)-\left(I-S_{n} \Theta_{n}^{M}\right) x_{n}
\end{aligned}
$$

that is,

$$
\begin{aligned}
(\mu A-\gamma V) x_{n}= & (\mu A-\gamma V)\left(I-W_{n}\right) x_{n} \\
& +\gamma\left(V u_{n}-V S_{n} u_{n}\right)-\frac{1}{\alpha_{n}}\left(I-W_{n}\right) x_{n} .
\end{aligned}
$$

Due to the nonexpansivity of $W_{n}$, we have that $I-W_{n}$ is monotone; that is, $\left\langle x-y,\left(I-W_{n}\right) x-\left(I-W_{n}\right) y\right\rangle \geq 0$, for all $x, y \in H$. Hence for any $p \in \Omega$,

$$
\begin{aligned}
\langle(\mu A & \left.-\gamma V) x_{n}, x_{n}-p\right\rangle \\
= & -\frac{1}{\alpha_{n}}\left\langle\left(I-W_{n}\right) x_{n}-\left(I-W_{n}\right) p, x_{n}-p\right\rangle \\
& +\left\langle(\mu A-\gamma V)\left(I-W_{n}\right) x_{n}, x_{n}-p\right\rangle \\
& +\gamma\left\langle V u_{n}-V S_{n} u_{n}, x_{n}-p\right\rangle \\
\leq & \left\langle(\mu A-\gamma V)\left(I-W_{n}\right) x_{n}, x_{n}-p\right\rangle \\
& +\gamma\left\|V u_{n}-V S_{n} u_{n}\right\|\left\|x_{n}-p\right\| \\
\leq & \left\langle(\mu A-\gamma V)\left(I-W_{n}\right) x_{n}, x_{n}-p\right\rangle \\
& +\gamma l\left\|u_{n}-S_{n} u_{n}\right\|\left\|x_{n}-p\right\| .
\end{aligned}
$$
obtain

Now replacing $n$ in (60) with $n_{j}$ and letting $j \rightarrow \infty$, we

$$
\begin{aligned}
\langle(\mu A & \left.-\gamma V) x^{*}, x^{*}-p\right\rangle \\
& =\lim _{j \rightarrow \infty}\left\langle(\mu A-\gamma V) x_{n_{j}}, x_{n_{j}}-p\right\rangle \\
& \leq \lim _{j \rightarrow \infty}\left\langle(\mu A-\gamma V)\left(x_{n_{j}}-S_{n_{j}} u_{n_{j}}\right), x_{n_{j}}-p\right\rangle=0 ;
\end{aligned}
$$

that is, $x^{*} \in \Omega$ is a solution of the variational inequality (27). 
Further, by the uniqueness of the solution of the variational inequality (27), we conclude that $x_{n} \rightarrow x^{*}$ as $n \rightarrow \infty$. We rewrite (27) as

$$
\left\langle(I-\mu A+\gamma V) x^{*}-x^{*}, x^{*}-p\right\rangle \geq 0, \quad \forall p \in \Omega .
$$

This is equivalent to the fixed point equation

$$
P_{\Omega}(I-\mu A+\gamma V) x^{*}=x^{*} .
$$

Theorem 13. Let $C$ be a nonempty closed convex subset of a real Hilbert space $H$ with $C \pm C \subseteq C$, and let $F_{k}, k \in$ $\{1,2, \ldots M\}$ be bifunctions from $C \times C$ to $\mathbb{R}$ which satisfies conditions (A1)-(A4). Let $f: C \rightarrow \mathbb{R}$ be a real-value convex function and $\nabla f$ a $\nu$-ism mapping with $\nu>0$. Assume the set $\Omega=U \cap \cap_{i=1}^{M} \operatorname{EP}\left(F_{k}\right) \neq \emptyset$. Let $V: C \rightarrow C$ is an l-Lipschitzian mapping with $l \geq 0$ and $A$ is a strongly positive bounded linear operator with coefficient $\bar{\gamma} \geq 0,0<\mu<2 \bar{\gamma} /\|A\|^{2}$ and $0<\gamma<\mu\left(\bar{\gamma}-\left(\mu\|A\|^{2} / 2\right)\right) / l=\tau /$ l. Given $x_{1} \in H$, let $\left\{x_{n}\right\}$ and $\left\{u_{n}\right\}$ be sequences generated by the following algorithm:

$$
\begin{gathered}
u_{n}=T_{r_{M, n}}^{F_{M}} T_{r_{M-1, n}}^{F_{M-1}} \cdots T_{r_{2, n}}^{F_{2}} T_{r_{1, n}}^{F_{1}} x_{n}, \\
x_{n+1}=\alpha_{n} \gamma V u_{n}+\left(I-\mu \alpha_{n} A\right) S_{n} u_{n},
\end{gathered}
$$

where $P_{C}\left(I-\lambda_{n} \nabla f\right)=\beta_{n} I+\left(1-\beta_{n}\right) S_{n}, \beta_{n}=\left(\left(2 \nu-\lambda_{n}\right) / 4 \nu\right)$, and $\lambda_{n} \in(0,2 \nu)$; if $\left\{\alpha_{n}\right\},\left\{\beta_{n}\right\}$, and $\left\{r_{k, n}\right\}$ satisfy the following conditions:

(i) $\left\{\alpha_{n}\right\} \subset(0,1), \lim _{n \rightarrow \infty} \alpha_{n}=0$ and $\sum_{n=1}^{\infty}\left|\alpha_{n+1}-\alpha_{n}\right|<$ $\infty$;

(ii) $\left\{r_{k, n}\right\} \subset(0, \infty), \liminf \operatorname{in}_{n \rightarrow \infty} r_{k, n}>0$ and $\sum_{n=1}^{\infty} \mid r_{k, n+1}-$ $r_{k, n} \mid<\infty$ for $k \in\{1,2, \ldots M\}$

(iii) $\left\{\beta_{n}\right\} \subset(0,(1 / 2)), \lim _{n \rightarrow \infty} \beta_{n}=0$ and $\sum_{n=1}^{\infty} \mid \beta_{n+1}-$ $\beta_{n} \mid<\infty$,

then, the sequence $\left\{x_{n}\right\}$ converges strongly to a point $x^{*} \in \Omega$, which solves the variational inequality (27).

Proof. The proof is divided into several steps.

Step 1. It shows first that $\left\{x_{n}\right\}$ is bounded.

Take any $p \in \Omega$, we have

$$
\left\|u_{n}-p\right\|=\left\|\Theta_{n}^{M} x_{n}-\Theta_{n}^{M} p\right\| \leq\left\|x_{n}-p\right\| .
$$

Thus, by (64), we derive that

$$
\begin{aligned}
\left\|x_{n+1}-p\right\| & \\
= & \left\|\alpha_{n} \gamma V u_{n}+\left(I-\mu \alpha_{n} A\right) S_{n} u_{n}-p\right\| \\
= & \|\left(I-\mu \alpha_{n} A\right) S_{n} u_{n}-\left(I-\mu \alpha_{n} A\right) S_{n} p \\
& \quad+\alpha_{n} \gamma V u_{n}-\alpha_{n} \gamma V p+\alpha_{n} \gamma V p-\alpha_{n} \mu A p \| \\
\leq & \left(1-\alpha_{n} \tau\right)\left\|u_{n}-p\right\|+\alpha_{n} \gamma l\left\|u_{n}-p\right\|+\alpha_{n}\|\gamma V p-\mu A p\| \\
\leq & \left(1-\alpha_{n}(\tau-\gamma l)\right)\left\|x_{n}-p\right\|+\alpha_{n}\|\gamma V p-\mu A p\| \\
= & \left(1-\alpha_{n}(\tau-\gamma l)\right)\left\|x_{n}-p\right\|+\alpha_{n}(\tau-\gamma l) \frac{\|\gamma V p-\mu A p\|}{\tau-\gamma l} \\
\leq & \max \left\{\left\|x_{n}-p\right\|, \frac{\|\gamma V p-\mu A p\|}{\tau-\gamma l}\right\} .
\end{aligned}
$$

By induction, we obtain $\left\|x_{n}-p\right\| \leq \max \left\{\left\|x_{1}-p\right\|,(\| \gamma V p-\right.$ $\mu A p \| /(\tau-\gamma l))\}, n \geq 1$. Hence, $\left\{x_{n}\right\}$ is bounded and so $\left\{u_{n}\right\}$. It follows from the Lipschitz continuity of $A, \nabla f$, and $V$ that $\left\{A x_{n}\right\},\left\{A u_{n}\right\}\left\{\nabla f\left(u_{n}\right)\right\}$, and $\left\{V u_{n}\right\}$ are also bounded. From the nonexpansivity of $S_{n}$, it follows that $\left\{S_{n} u_{n}\right\}$ is also bounded.

Step 2. It shows that

$$
\left\|x_{n+1}-x_{n}\right\| \longrightarrow 0
$$

By (68), we have

$$
\begin{aligned}
\| x_{n+1} & -x_{n} \| \\
= & \| \alpha_{n} \gamma V u_{n}+\left(I-\mu \alpha_{n} A\right) S_{n} u_{n} \\
& -\alpha_{n-1} \gamma V u_{n-1}-\left(I-\mu \alpha_{n-1} A\right) S_{n-1} u_{n-1} \| \\
\leq & \alpha_{n} \gamma\left\|V u_{n}-V u_{n-1}\right\|+\left|\alpha_{n}-\alpha_{n-1}\right| \gamma\left\|V u_{n-1}\right\| \\
& +\left\|\left(I-\mu \alpha_{n} A\right) S_{n} u_{n}-\left(I-\mu \alpha_{n} A\right) S_{n} u_{n-1}\right\| \\
& +\left\|\left(I-\mu \alpha_{n} A\right) S_{n} u_{n-1}-\left(I-\mu \alpha_{n} A\right) S_{n-1} u_{n-1}\right\| \\
& +\left\|\left(I-\mu \alpha_{n} A\right) S_{n-1} u_{n-1}-\left(I-\mu \alpha_{n-1} A\right) S_{n-1} u_{n-1}\right\| \\
\leq & \alpha_{n} \gamma l\left\|u_{n}-u_{n-1}\right\|+\left|\alpha_{n}-\alpha_{n-1}\right| \\
& \times\left(\left\|\gamma V u_{n-1}\right\|+\left\|\mu A S_{n-1} u_{n-1}\right\|\right) \\
& +\left(1-\alpha_{n} \tau\right)\left(\left\|u_{n}-u_{n-1}\right\|+\left\|S_{n} u_{n-1}-S_{n-1} u_{n-1}\right\|\right) \\
\leq & \left(1-\alpha_{n}(\tau-\gamma l)\right)\left\|u_{n}-u_{n-1}\right\| \\
& +\left|\alpha_{n}-\alpha_{n-1}\right|\left(\left\|\gamma V u_{n-1}\right\|+\left\|\mu A S_{n-1} u_{n-1}\right\|\right) \\
& +\left(1-\alpha_{n} \tau\right)\left\|S_{n} u_{n-1}-S_{n-1} u_{n-1}\right\| .
\end{aligned}
$$

Next we estimate $\left\|S_{n} u_{n-1}-S_{n-1} u_{n-1}\right\|$. 
8

Journal of Applied Mathematics

Observe that

$$
\begin{aligned}
& \left\|S_{n} u_{n-1}-S_{n-1} u_{n-1}\right\| \\
& =\| \frac{P_{C}\left(I-\lambda_{n} \nabla f\right)-\beta_{n} I}{1-\beta_{n}} u_{n-1} \\
& -\frac{P_{C}\left(I-\lambda_{n-1} \nabla f\right)-\beta_{n-1} I}{1-\beta_{n-1}} u_{n-1} \| \\
& =\| \frac{4 v P_{C}\left(I-\lambda_{n} \nabla f\right)-\left(2 v-\lambda_{n}\right) I}{2 v+\lambda_{n}} u_{n-1} \\
& -\frac{4 v P_{C}\left(I-\lambda_{n-1} \nabla f\right)-\left(2 v-\lambda_{n-1}\right) I}{2 \nu+\lambda_{n-1}} u_{n-1} \| \\
& \leq \| \frac{4 v P_{C}\left(I-\lambda_{n} \nabla f\right)}{2 \nu+\lambda_{n}} u_{n-1} \\
& -\frac{4 \nu P_{\mathrm{C}}\left(I-\lambda_{n-1} \nabla f\right)}{2 \nu+\lambda_{n-1}} u_{n-1} \| \\
& +\left\|\frac{2 v-\lambda_{n-1}}{2 v+\lambda_{n-1}} u_{n-1}-\frac{2 v-\lambda_{n}}{2 v+\lambda_{n}} u_{n-1}\right\| \\
& =\|\left(4 v\left(2 v+\lambda_{n-1}\right) P_{C}\left(I-\lambda_{n} \nabla f\right) u_{n-1}\right. \\
& \left.-4 v\left(2 v+\lambda_{n}\right) P_{C}\left(I-\lambda_{n-1} \nabla f\right) u_{n-1}\right) \\
& \times\left(\left(2 v+\lambda_{n}\right)\left(2 v+\lambda_{n-1}\right)\right)^{-1} \| \\
& +\frac{4 \nu\left|\lambda_{n}-\lambda_{n-1}\right|}{\left(2 \nu+\lambda_{n}\right)\left(2 \nu+\lambda_{n-1}\right)}\left\|u_{n-1}\right\| \\
& \leq \|\left(4 \nu\left(\lambda_{n-1}-\lambda_{n}\right) P_{C}\left(I-\lambda_{n} \nabla f\right) u_{n-1}+4 \nu\left(2 \nu+\lambda_{n}\right)\right. \\
& \left.\times\left(P_{C}\left(I-\lambda_{n} \nabla f\right) u_{n-1}-P_{C}\left(I-\lambda_{n-1} \nabla f\right) u_{n-1}\right)\right) \\
& \times\left(\left(2 v+\lambda_{n}\right)\left(2 v+\lambda_{n-1}\right)\right)^{-1} \| \\
& +\frac{4 \nu\left|\lambda_{n}-\lambda_{n-1}\right|}{\left(2 \nu+\lambda_{n}\right)\left(2 \nu+\lambda_{n-1}\right)}\left\|u_{n-1}\right\| \\
& \leq \frac{4 \nu\left|\lambda_{n-1}-\lambda_{n}\right|\left\|P_{C}\left(I-\lambda_{n} \nabla f\right) u_{n-1}\right\|}{\left(2 \nu+\lambda_{n}\right)\left(2 \nu+\lambda_{n-1}\right)} \\
& +\frac{4 \nu\left\|P_{C}\left(I-\lambda_{n} \nabla f\right) u_{n-1}-P_{C}\left(I-\lambda_{n-1} \nabla f\right) u_{n-1}\right\|}{2 \nu+\lambda_{n-1}} \\
& +\frac{4 \nu\left|\lambda_{n}-\lambda_{n-1}\right|}{\left(2 v+\lambda_{n}\right)\left(2 v+\lambda_{n-1}\right)}\left\|u_{n-1}\right\| \\
& \leq\left|\lambda_{n}-\lambda_{n-1}\right|\left(\frac{1}{\nu}\left\|P_{C}\left(I-\lambda_{n} \nabla f\right) u_{n-1}\right\|\right. \\
& \left.+2\left\|\nabla f\left(u_{n-1}\right)\right\|+\frac{1}{v}\left\|u_{n-1}\right\|\right)
\end{aligned}
$$

$$
\begin{aligned}
& =4 \nu\left|\beta_{n}-\beta_{n-1}\right|\left(\frac{1}{\nu}\left\|P_{C}\left(I-\lambda_{n} \nabla f\right) u_{n-1}\right\|\right. \\
& \left.+2\left\|\nabla f\left(u_{n-1}\right)\right\|+\frac{1}{\nu}\left\|u_{n-1}\right\|\right) \\
& \leq M_{1}\left|\beta_{n}-\beta_{n-1}\right|,
\end{aligned}
$$

where $M_{1}=\sup _{n}\left\{4\left\|P_{C}\left(I-\lambda_{n} \nabla f\right) u_{n-1}\right\|+8 \nu\left\|\nabla f\left(u_{n-1}\right)\right\|+\right.$ $\left.4\left\|u_{n-1}\right\|\right\}$.

Substitute (69) into (68), we get

$$
\begin{aligned}
\| x_{n+1} & -x_{n} \| \\
\leq & \left(1-\alpha_{n}(\tau-\gamma l)\right)\left\|u_{n}-u_{n-1}\right\| \\
& +\left|\alpha_{n}-\alpha_{n-1}\right|\left(\left\|\gamma V u_{n-1}\right\|+\left\|\mu A S_{n-1} u_{n-1}\right\|\right) \\
& +M_{1}\left|\beta_{n}-\beta_{n-1}\right| \\
\leq & \left(1-\alpha_{n}(\tau-\gamma l)\right)\left\|u_{n}-u_{n-1}\right\| \\
& +\left(\left|\alpha_{n}-\alpha_{n-1}\right|+\left|\beta_{n}-\beta_{n-1}\right|\right) \\
& \times\left(\left\|\gamma V u_{n-1}\right\|+\left\|\mu A S_{n-1} u_{n-1}\right\|+M_{1}\right) \\
\leq & \left(1-\alpha_{n}(\tau-\gamma l)\right)\left\|u_{n}-u_{n-1}\right\| \\
& +M_{2}\left(\left|\alpha_{n}-\alpha_{n-1}\right|+\left|\beta_{n}-\beta_{n-1}\right|\right),
\end{aligned}
$$

for some appropriate positive constant $M_{2}$ such that

$$
M_{2}=\sup _{n}\left\{\left\|\gamma V u_{n-1}\right\|+\left\|\mu A S_{n-1} u_{n-1}\right\|+M_{1}\right\} .
$$

Observe that

$$
\begin{aligned}
& \| u_{n+1}-u_{n} \| \\
&=\left\|\Theta_{n+1}^{M} x_{n+1}-\Theta_{n}^{M} x_{n}\right\| \\
& \leq\left\|\Theta_{n+1}^{M} x_{n+1}-\Theta_{n+1}^{M} x_{n}\right\| \\
&+\left\|\Theta_{n+1}^{M} x_{n}-\Theta_{n}^{M} x_{n}\right\| \\
& \leq\left\|x_{n+1}-x_{n}\right\| \\
&+\left\|\Theta_{n+1}^{M} x_{n}-\Theta_{n}^{M} x_{n}\right\| \\
& \leq\left\|x_{n+1}-x_{n}\right\| \\
&+\left\|T_{r_{M, n+1}}^{F_{M}} \Theta_{n+1}^{M-1} x_{n}-T_{r_{M, n}}^{F_{M}} \Theta_{n+1}^{M-1} x_{n}\right\| \\
&+\left\|T_{r_{M, n}}^{F_{M}} \Theta_{n+1}^{M-1} x_{n}-T_{r_{M, n}}^{F_{M}} T_{r_{M-1, n}}^{F_{M-1}} \Theta_{n+1}^{M-2} x_{n}\right\| \\
&+\cdots+\left\|T_{r_{M, n}}^{F_{M}} T_{r_{M-1, n}}^{F_{M-1}} \ldots \Theta_{n+1}^{2} x_{n}-T_{r_{M, n}}^{F_{M}} \ldots T_{r_{2, n}}^{F_{2}} T_{r_{1, n+1}}^{F_{1}} x_{n}\right\| \\
&+\left\|T_{r_{M, n}}^{F_{M}} \ldots T_{r_{2, n}}^{F_{2}} T_{r_{1, n+1}}^{F_{1}} x_{n}-u_{n}\right\| \\
& \leq\left\|x_{n+1}-x_{n}\right\|+\left\|T_{r_{M, n+1}}^{F_{M}} \Theta_{n+1}^{M-1} x_{n}-T_{r_{M, n}}^{F_{M}} \Theta_{n+1}^{M-1} x_{n}\right\| \\
&+\left\|\Theta_{n+1}^{M-1} x_{n}-T_{r_{M-1, n}}^{F_{M-1}} \Theta_{n+1}^{M-2} x_{n}\right\| \\
&+\cdots+\left\|T_{r_{1, n+1}}^{F_{1}} x_{n}-T_{r_{1, n}}^{F_{1}} x_{n}\right\| . \\
& F_{r_{1, n+1}} x_{n}-T_{r_{2, n}}^{F_{2}} T_{r_{1, n+1}}^{F_{1}} x_{n} \| \\
& F_{n} \|
\end{aligned}
$$


By Lemma 10, we get

$$
\begin{aligned}
& \left\|u_{n+1}-u_{n}\right\| \\
& \leq\left\|x_{n+1}-x_{n}\right\| \\
& +\frac{\left|r_{M, n+1}-r_{M, n}\right|}{r_{M, n+1}}\left\|T_{r_{M, n+1}}^{F_{M}} \Theta_{n+1}^{M-1} x_{n}-\Theta_{n+1}^{M-1} x_{n}\right\| \\
& +\frac{\left|r_{M-1, n+1}-r_{M-1, n}\right|}{r_{M-1, n+1}}\left\|T_{r_{M-1, n}}^{F_{M-1}} \Theta_{n+1}^{M-2} x_{n}-\Theta_{n+1}^{M-2} x_{n}\right\| \\
& +\cdots+\frac{\left|r_{2, n+1}-r_{2, n}\right|}{r_{2, n+1}}\left\|T_{r_{2, n+1}}^{F_{2}} T_{r_{1, n+1}}^{F_{1}} x_{n}-T_{r_{1, n+1}}^{F_{1}} x_{n}\right\| \\
& +\frac{\left|r_{1, n+1}-r_{1, n}\right|}{r_{1, n+1}}\left\|T_{r_{1, n+1}}^{F_{1}} x_{n}-x_{n}\right\| .
\end{aligned}
$$

Combing (70) and (73), we have

$$
\begin{aligned}
\| x_{n+1} & -x_{n} \| \\
\leq & \left(1-\alpha_{n}(\tau-\gamma l)\right)\left\|x_{n}-x_{n-1}\right\| \\
& +\frac{\left|r_{M, n}-r_{M, n-1}\right|}{r_{M, n}}\left\|T_{r_{M, n}}^{F_{M}} \Theta_{n}^{M-1} x_{n-1}-\Theta_{n}^{M-1} x_{n-1}\right\| \\
& +\frac{\left|r_{M-1, n}-r_{M-1, n-1}\right|}{r_{M-1, n}} \\
& \times \mid T_{r_{M-1, n-1}}^{F_{M-1}} \Theta_{n-2}^{M} x_{n-1}-\Theta_{n}^{M-2} x_{n-1} \| \\
& +\cdots+\frac{\left|r_{2, n}-r_{2, n-1}\right|}{r_{2, n}}\left\|T_{r_{2, n}}^{F_{2}} T_{r_{1, n}}^{F_{1}} x_{n-1}-T_{r_{1, n}}^{F_{1}} x_{n-1}\right\| \\
& +\frac{\left|r_{1, n}-r_{1, n-1}\right|}{r_{1, n}}\left\|T_{r_{1, n}}^{F_{1}} x_{n-1}-x_{n-1}\right\| \\
& +M_{2}\left(\left|\alpha_{n}-\alpha_{n-1}\right|+\left|\beta_{n}-\beta_{n-1}\right|\right) .
\end{aligned}
$$

By Lemma 2, It follows from conditions (i)-(iii) that (67) holds. Further from (73), we have

$$
\left\|u_{n+1}-u_{n}\right\| \longrightarrow 0
$$

Step 3. It shows that

$$
\left\|x_{n}-S_{n} x_{n}\right\| \longrightarrow 0
$$

For any $p \in \Omega$, as the same proof of Theorem 12, we have

$$
\left\|u_{n}-p\right\|^{2} \leq\left\|x_{n}-p\right\|^{2}-\left\|\Theta_{n}^{k} x_{n}-\Theta_{n}^{k-1} x_{n}\right\|^{2} .
$$

Then from (64) and (77), we derive that

$$
\begin{aligned}
& \left\|x_{n+1}-p\right\|^{2} \\
& \quad=\left\|\alpha_{n} \gamma V u_{n}+\left(I-\mu \alpha_{n} A\right) S_{n} u_{n}-p\right\|^{2} \\
& \quad=\left\|\left(I-\mu \alpha_{n} A\right) S_{n} u_{n}-\left(I-\mu \alpha_{n} A\right) S_{n} p+\alpha_{n} \gamma V u_{n}-\alpha_{n} \mu A p\right\|^{2}
\end{aligned}
$$

$$
\begin{aligned}
\leq & \left(1-\alpha_{n} \tau\right)^{2}\left\|u_{n}-p\right\|^{2} \\
& +2\left(1-\alpha_{n} \tau\right) \alpha_{n}\left\|u_{n}-p\right\|\left\|\gamma V u_{n}-\mu A p\right\| \\
& +\alpha_{n}^{2}\left\|\gamma V u_{n}-\mu A p\right\|^{2} \\
\leq & \left\|x_{n}-p\right\|^{2}-\left\|\Theta_{n}^{k} x_{n}-\Theta_{n}^{k-1} x_{n}\right\|^{2} \\
& +2\left(1-\alpha_{n} \tau\right) \alpha_{n}\left\|u_{n}-p\right\|\left\|\gamma V u_{n}-\mu A p\right\| \\
& +\alpha_{n}^{2}\left\|\gamma V u_{n}-\mu A p\right\|^{2} .
\end{aligned}
$$

From $\alpha_{n} \rightarrow 0$ and (67), we have

$$
\left\|\Theta_{n}^{k} x_{n}-\Theta_{n}^{k-1} x_{n}\right\|^{2} \longrightarrow 0
$$

Further we have

$$
\left\|x_{n}-u_{n}\right\| \longrightarrow 0
$$

Next,

$$
\begin{aligned}
\| x_{n}- & S_{n} x_{n} \| \\
& =\left\|x_{n}-x_{n+1}+x_{n+1}-S_{n} u_{n}+S_{n} u_{n}-S_{n} x_{n}\right\| \\
& \leq\left\|x_{n}-x_{n+1}\right\|+\left\|x_{n+1}-S_{n} u_{n}\right\|+\left\|S_{n} u_{n}-S_{n} x_{n}\right\| \\
& \leq\left\|x_{n}-x_{n+1}\right\|+\alpha_{n}\left\|\gamma V u_{n}-\mu A S_{n} u_{n}\right\|+\left\|u_{n}-x_{n}\right\| .
\end{aligned}
$$

It follows from (67) and (80) that (76) holds. Further we have $\left\|u_{n}-S_{n} u_{n}\right\| \rightarrow 0$.

Step 4. It shows that

$$
\limsup _{n \rightarrow \infty}\left\langle(\gamma V-\mu A) x^{*}, x_{n}-x^{*}\right\rangle \leq 0
$$

where $x^{*}=P_{\Omega}(I-\mu A+\gamma V) x^{*}$ is a unique solution of the variational inequality (27). Indeed, take a subsequence $\left\{x_{n_{j}}\right\}$ of $\left\{x_{n}\right\}$ such that

$$
\begin{aligned}
\limsup _{n \rightarrow \infty} & \left\langle(\gamma V-\mu A) x^{*}, x_{n}-x^{*}\right\rangle \\
& =\lim _{j \rightarrow \infty}\left\langle(\gamma V-\mu A) x^{*}, x_{n_{j}}-x^{*}\right\rangle .
\end{aligned}
$$

Since $\left\{x_{n_{j}}\right\}$ is bounded, there exists a subsequence $\left\{x_{n_{j_{k}}}\right\}$ of $\left\{x_{n_{j}}\right\}$ which converges weakly to $q$. Without loss of generality, we can assume $x_{n_{j}} \rightarrow q$. By the same argument as in the proof of Theorem 12, we have $q \in \Omega$. Since $x^{*}=P_{\Omega}(I-\mu A+\gamma V) x^{*}$, it follows that

$$
\begin{aligned}
\limsup _{n \rightarrow \infty} & \left\langle(\gamma V-\mu A) x^{*}, x_{n}-x^{*}\right\rangle \\
& =\lim _{j \rightarrow \infty}\left\langle(\gamma V-\mu A) x^{*}, x_{n_{j}}-x^{*}\right\rangle \\
& =\left\langle(\gamma V-\mu A) x^{*}, q-x^{*}\right\rangle \leq 0 .
\end{aligned}
$$


Step 5. It shows that

$$
x_{n} \longrightarrow x^{*}
$$

Consider

$$
\begin{aligned}
\langle(\gamma V & \left.-\mu A) x^{*}, x_{n+1}-x^{*}\right\rangle \\
= & \left\langle(\gamma V-\mu A) x^{*}, x_{n+1}-x_{n}\right\rangle \\
& +\left\langle(\gamma V-\mu A) x^{*}, x_{n}-x^{*}\right\rangle \\
\leq & \left\|(\gamma V-\mu A) x^{*}\right\|\left\|x_{n+1}-x_{n}\right\| \\
& +\left\langle(\gamma V-\mu A) x^{*}, x_{n}-x^{*}\right\rangle .
\end{aligned}
$$

It follows from (67) and (82) that

$$
\begin{aligned}
& \limsup _{n \rightarrow \infty}\left\langle(\gamma V-\mu A) x^{*}, x_{n+1}-x^{*}\right\rangle \leq 0, \\
&\left\|x_{n+1}-x^{*}\right\|^{2} \\
&=\left\|\alpha_{n} \gamma V u_{n}+\left(I-\mu \alpha_{n} A\right) S_{n} u_{n}-x^{*}\right\|^{2} \\
&=\|\left(I-\mu \alpha_{n} A\right) S_{n} u_{n}-\left(I-\mu \alpha_{n} A\right) x^{*} \\
& \quad+\alpha_{n}\left(\gamma V u_{n}-\mu A x^{*}\right) \|^{2} \\
& \leq\left\|\left(I-\mu \alpha_{n} A\right) S_{n} u_{n}-\left(I-\mu \alpha_{n} A\right) x^{*}\right\|^{2} \\
& \quad+2 \alpha_{n}\left\langle\gamma V u_{n}-\mu A x^{*}, x_{n+1}-x^{*}\right\rangle \\
& \leq\left(1-\alpha_{n} \tau\right)^{2}\left\|u_{n}-x^{*}\right\|^{2} \\
&+ 2 \alpha_{n}\left\langle\gamma V u_{n}-\gamma V x^{*}, x_{n+1}-x^{*}\right\rangle \\
&+2 \alpha_{n}\left\langle(\gamma V-\mu A) x^{*}, x_{n+1}-x^{*}\right\rangle \\
& \leq\left(1-\alpha_{n} \tau\right)^{2}\left\|x_{n}-x^{*}\right\|^{2} \\
&+\alpha_{n} \gamma l\left(\left\|x_{n}-x^{*}\right\|^{2}+\left\|x_{n+1}-x^{*}\right\|^{2}\right) \\
&+2 \alpha_{n}\left\langle(\gamma V-\mu A) x^{*}, x_{n+1}-x^{*}\right\rangle .
\end{aligned}
$$

This implies that

$$
\begin{aligned}
&\left\|x_{n+1}-x^{*}\right\|^{2} \\
& \leq \frac{\left(1-\alpha_{n} \tau\right)^{2}+\alpha_{n} \gamma l}{1-\alpha_{n} \gamma l}\left\|x_{n}-x^{*}\right\|^{2} \\
&+\frac{2 \alpha_{n}}{1-\alpha_{n} \gamma l}\left\langle(\gamma V-\mu A) x^{*}, x_{n+1}-x^{*}\right\rangle \\
& \leq\left(1-\frac{2 \alpha_{n}(\tau-\gamma l)}{1-\alpha_{n} \alpha \gamma}\right)\left\|x_{n}-x^{*}\right\|^{2} \\
&+\frac{2 \alpha_{n}}{1-\alpha_{n} \gamma l}\left\langle(\gamma V-\mu A) x^{*}, x_{n+1}-x^{*}\right\rangle+\frac{\left(\alpha_{n} \tau\right)^{2}}{1-\alpha_{n} \gamma l} M_{3},
\end{aligned}
$$

where $M_{3}=\sup _{n}\left\|x_{n}-x^{*}\right\|^{2}, n \geq 1$. It is easily to see that $\gamma_{n}=$ $\left(\left(2 \alpha_{n}(\tau-\gamma l)\right) /\left(1-\alpha_{n} \gamma l\right)\right)$. Hence by Lemma 2 , the sequence $\left\{x_{n}\right\}$ converges strongly to $x^{*}$.

Remark 14. If $M=1, \mu=1$, then Theorem 13 reduces to Theorem 3.2 of Tian and Liu [9].
TABLE 1: $x^{(1)}=(0,0)^{\top}$ (initial guess).

\begin{tabular}{lccc}
\hline$n$ (iterative number) & $x^{(n)}$ & errors $(n)$ & $f^{(n)}$ \\
\hline 2262 & $(1.9986,1.9979)$ & $2.5 \times 10^{-3}$ & -1.9986 \\
22627 & $(1.9999,1.9998)$ & $2.479 \times 10^{-4}$ & -1.9999 \\
226274 & $(2.0000,2.0000)$ & $2.478 \times 10^{-5}$ & -2.0000 \\
\hline
\end{tabular}

\section{Numerical Result}

In this section, we consider the following simple example to demonstrate the effectiveness, realization and convergence of the algorithm in Theorem 13.

Let $R^{2}$ be the two dimensional Euclidean space with usual inner product $\left\langle x^{(1)}, x^{(2)}\right\rangle=x_{1}^{(1)} x_{1}^{(2)}+x_{2}^{(1)} x_{2}^{(2)},\left(\forall x^{(1)}=\right.$ $\left.\left(x_{1}^{(1)}, x_{2}^{(1)}\right)^{\top}, x^{(2)}=\left(x_{1}^{(2)}, x_{2}^{(2)}\right)^{\top} \in R^{2}\right)$ and induced norm $\|x\|=\sqrt{x_{1}^{2}+x_{2}^{2}}\left(\forall x=\left(x_{1}, x_{2}\right)^{\top} \in R^{2}\right)$. Next, we consider the following simple example.

Example 15. Consider the following constrained convex minimization problem:

$$
\begin{array}{ll}
\operatorname{minimize} & f(x)=x_{1}^{2}+\frac{1}{2} x_{2}^{2}-x_{1} x_{2}-2 x_{1}, \quad x \in R^{2}, \\
\text { s.t. } & x_{1} \geq 0, \quad x_{2} \geq 0 .
\end{array}
$$

In Theorem 13, let $H=R^{2}, C=[0, \infty) \times[0, \infty)$, and $F_{k} \equiv 0$, for all $x, y \in C, k \in\{1,2, \ldots, M\}$. Give $A=I, V x=2 x$, for all $x \in H$, with Lipschitz coefficient $l=2$.

Compute the gradient of $f$, we have $\nabla f(x)=\left(2 x_{1}-x_{2}-\right.$ $\left.2, x_{2}-x_{1}\right)^{\top}$ is $(1 / 3)$-ism and $\nabla f^{2}(x)=\left(\begin{array}{cc}2 & -1 \\ -1 & 1\end{array}\right)$ is positively definite; thus $f$ is convex. Give the parameters $\alpha_{n}=(1 / n)$, $\beta_{n}=(1 / 4 n)$ for every $n \geq 1$, fix $\mu=1$, and $\gamma=(1 / 10)$. Then $\left\{x^{(n)}\right\}$ is the sequence generated by

$$
x^{(n+1)}=\frac{1}{5 n} x^{(n)}+\frac{n-1}{n} S_{n} x^{(n)},
$$

where

$$
S_{n} x^{(n)}=\frac{P_{C}\left(I-\lambda_{n} \nabla f\right) x^{(n)}-\beta_{n} x^{(n)}}{1-\beta_{n}} .
$$

As $n \rightarrow \infty$, we have $\left\{x^{(n)}\right\} \rightarrow x^{*}=(2,2)^{\top}, f\left(x^{*}\right)=-2$.

Take the initial guess $x^{(1)}=(0,0)^{\top}$, we obtain the numerical results as shown in Table 1 .

\section{Acknowledgments}

The author would like to thank the referee for valuable suggestions to improve the paper and the Fundamental Research Funds for the Central Universities (Grant: 3122013k004).

\section{References}

[1] P. L. Combettes and S. A. Hirstoaga, "Equilibrium programming in Hilbert spaces," Journal of Nonlinear and Convex Analysis, vol. 6, no. 1, pp. 117-136, 2005. 
[2] Y. Liu, "A general iterative method for equilibrium problems and strict pseudo-contractions in Hilbert spaces," Nonlinear Analysis. Theory, Methods \& Applications A, vol. 71, no. 10, pp. 48524861, 2009.

[3] G. Marino and H.-K. Xu, "Weak and strong convergence theorems for strict pseudo-contractions in Hilbert spaces," Journal of Mathematical Analysis and Applications, vol. 329, no. 1, pp. 336-346, 2007.

[4] S. Takahashi and W. Takahashi, "Strong convergence theorem for a generalized equilibrium problem and a nonexpansive mapping in a Hilbert space," Nonlinear Analysis. Theory, Methods \& Applications A, vol. 69, no. 3, pp. 1025-1033, 2008.

[5] V. Colao, G. L. Acedo, and G. Marino, "An implicit method for finding common solutions of variational inequalities and systems of equilibrium problems and fixed points of infinite family of nonexpansive mappings," Nonlinear Analysis. Theory, Methods \& Applications A, vol. 71, no. 7-8, pp. 2708-2715, 2009.

[6] P. Duan, "Convergence theorems concerning hybrid methods for strict pseudocontractions and systems of equilibrium problems," Journal of Inequalities and Applications, vol. 2010, Article ID 396080, 14 pages, 2010.

[7] H. He, S. Liu, and Y. J. Cho, "An explicit method for systems of equilibrium problems and fixed points of infinite family of nonexpansive mappings," Journal of Computational and Applied Mathematics, vol. 235, no. 14, pp. 4128-4139, 2011.

[8] L.-C. Ceng, Q. H. Ansari, and J.-C. Yao, "Some iterative methods for finding fixed points and for solving constrained convex minimization problems," Nonlinear Analysis. Theory, Methods \& Applications A, vol. 74, no. 16, pp. 5286-5302, 2011.

[9] M. Tian and L. Liu, "General iterative methods for equilibrium and constrained convex minimization problem," Optimazation, 2012.

[10] H. K. Xu, "An iterative approach to quadratic optimization," Journal of Optimization Theory and Applications, vol. 116, no. 3, pp. 659-678, 2003.

[11] H.-K. Xu, "Averaged mappings and the gradient-projection algorithm," Journal of Optimization Theory and Applications, vol. 150, no. 2, pp. 360-378, 2011.

[12] M. Su and H.-K. Xu, "Remarks on the gradient-projection algorithm," Journal of Nonlinear Analysis and Optimization, vol. 1, no. 1, pp. 35-43, 2010.

[13] S. Wang, "A general iterative method for obtaining an infinite family of strictly pseudo-contractive mappings in Hilbert spaces," Applied Mathematics Letters of Rapid Publication, vol. 24, no. 6, pp. 901-907, 2011.

[14] E. Blum and W. Oettli, "From optimization and variational inequalities to equilibrium problems," The Mathematics Student, vol. 63, no. 1-4, pp. 123-145, 1994. 


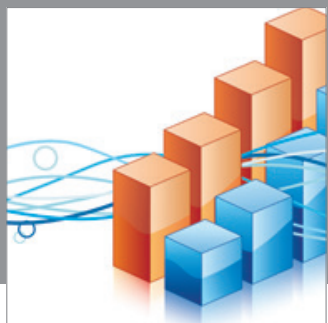

Advances in

Operations Research

mansans

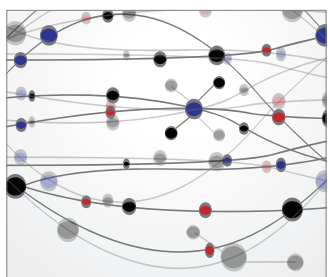

The Scientific World Journal
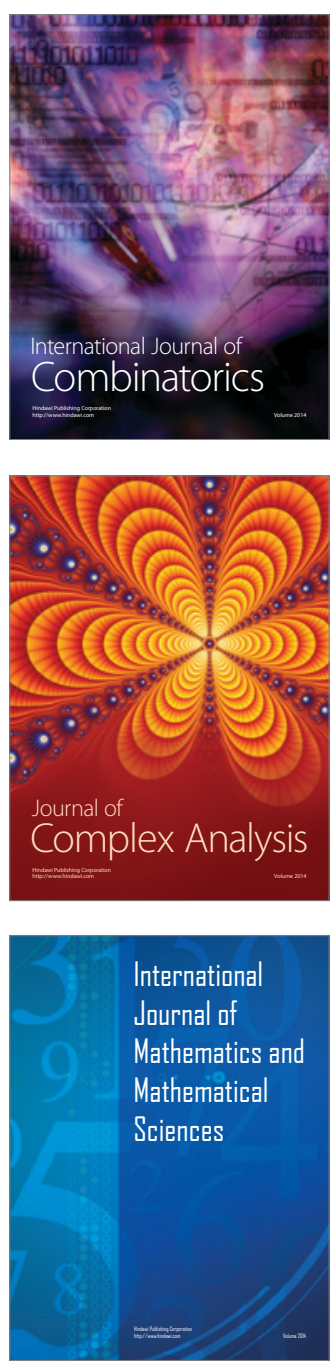
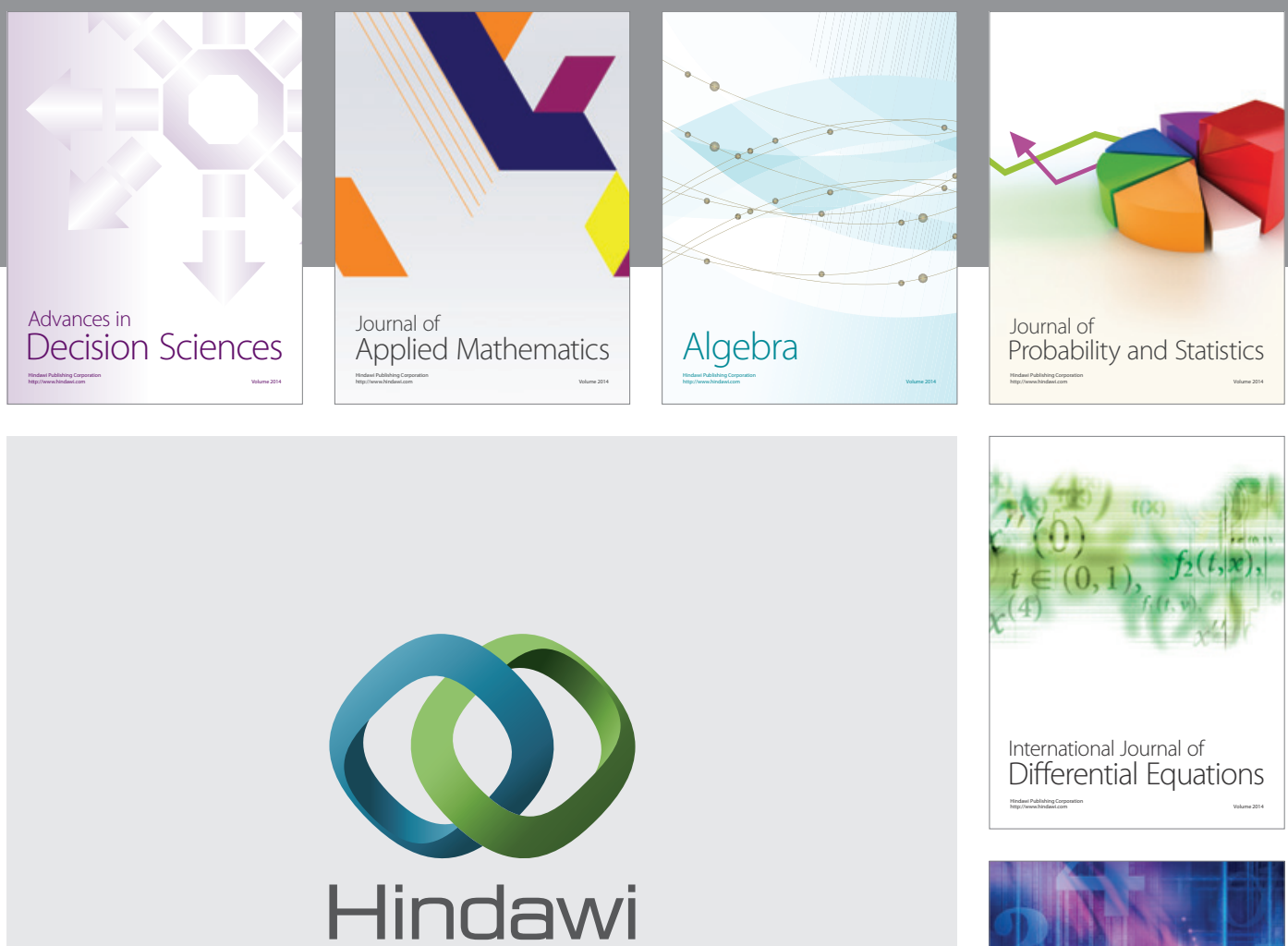

Submit your manuscripts at http://www.hindawi.com
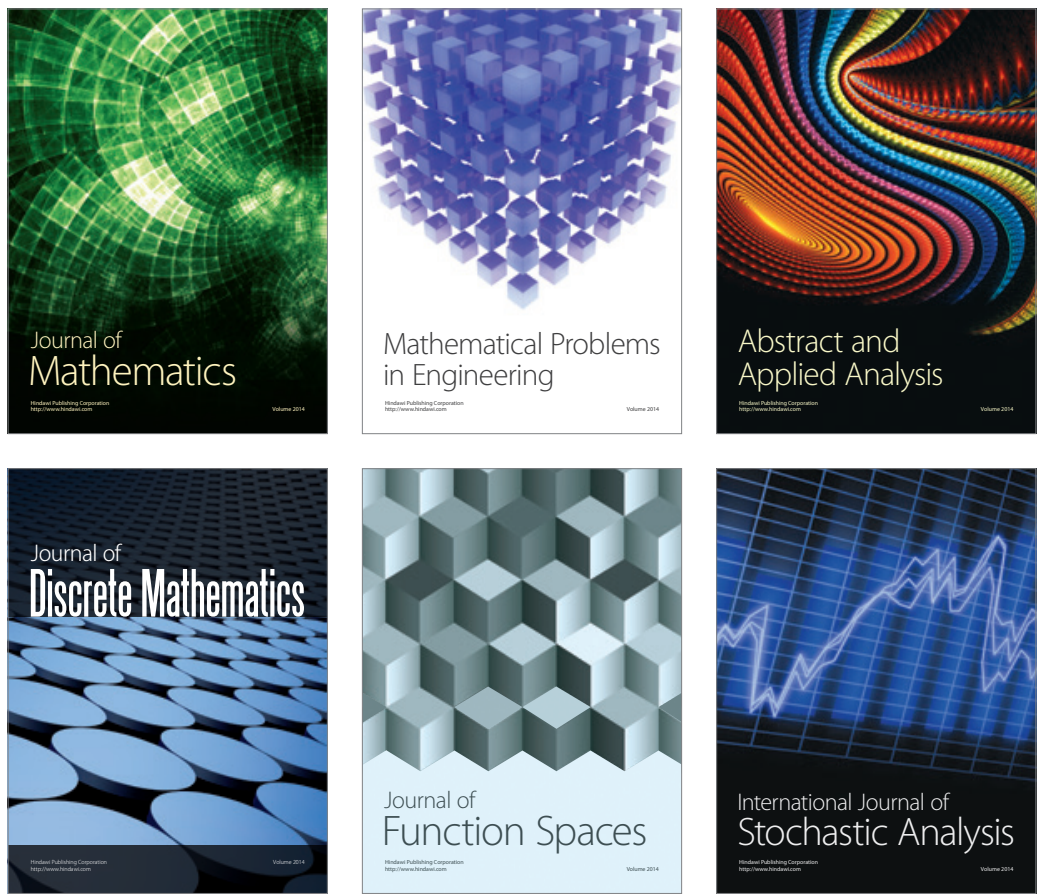

Journal of

Function Spaces

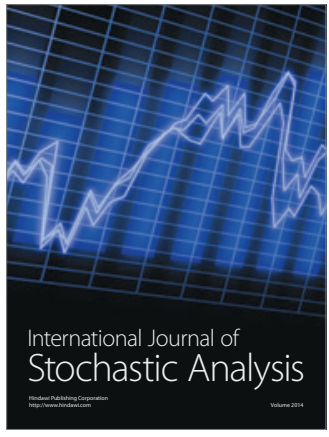

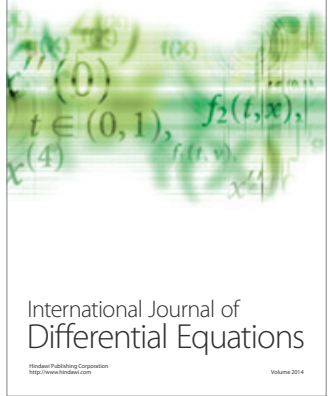
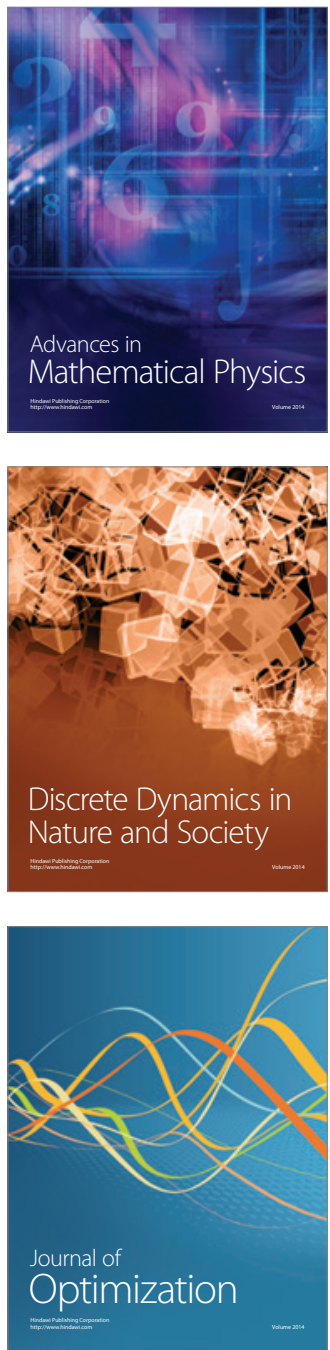